\title{
Closed-Loop INS/TACAN/ALT Positioning System
}

\begin{abstract}
P. KANIEWSKI*
Military University of Technology, Gen. S. Kaliskiego 2, 00-908 Warsaw, Poland

The paper presents a design of aircraft integrated positioning system, composed of INS, TACAN and altimeter (ALT). This paper describes a modified, closed-loop version of INS/TACAN/ALT system and compares it to the previous design. The system is integrated according to the scheme of compensation with feed-backward INS correction and it employs a complementary extended Kalman filter. The paper includes chosen results of simulations obtained for the open-loop and closed-loop INS/TACAN/ALT systems and discusses advantages of the new design.
\end{abstract}

PACS numbers: 02.70.-c, 07.05.Tp, 07.57.-c, 89.40.Dd

\section{Introduction}

Contemporary positioning systems are usually composed of several navigation devices and an algorithm of joint data processing (navigation filter), which is often a Kalman filter [1-3]. In aircraft positioning and navigation, INS/GNSS integrated systems are frequently applied [4]. Integration of inertial navigation systems (INS) and global navigation satellite system (GNSS) receivers via the Kalman filter presents one of the best achievements in positioning technology and one of the most successful applications of the Kalman filter. Although integration of INS and GNSS is very common, it is not the only possible option. Similar advantages can be gained in integrated positioning systems composed of INS and other than GNSS receiver radiotechnical devices. The presented in this paper INS/TACAN/ALT positioning system, composed of INS, Tactical Air Navigation System (TACAN) [5] and altimeter (ALT) [5], is an example of such an alternative solution.

\section{Measurements in INS/TACAN/ALT system}

The INS contains accelerometers and gyros, enabling calculation of linear and angular displacement [6]. Velocity and position of the vehicle are obtained through

${ }^{*}$ e-mail: pkaniewski@wat.edu.pl 
initialized single and double integration of accelerations, previously transformed from the body frame to the navigation frame of Refs. $[4,6]$. It is assumed here that INS position $(x, y, z)^{\text {INS }}$ will be expressed in an Earth-fixed reference frame $O X Y Z$, with its origin at the location of TACAN ground station and with axes coinciding with the axes of the local horizontal frame of reference ENU (East-North-Up). The assumed frame is suitable for short-range systems, containing a navigation device, which measurements are to be referenced to a fixed point on the Earth surface [6], as is the case in INS/TACAN/ALT system.

TACAN provides for measurements of distance $D$ between the aircraft on-board equipment and the ground station as well as the azimuth $\theta$ of aircraft with respect to the ground station. The ALT provides for the altitude $H$ of aircraft above the horizontal plane $O X Y$.

The relationship between the measurements and the coordinates of aircraft position is illustrated in Fig. 1. As one can see, the relationship between the altitude $H$ and the coordinate $z$ is linear, whereas the distance $D$ and azimuth $\theta$ from TACAN are non-linearly related to the aircraft position.

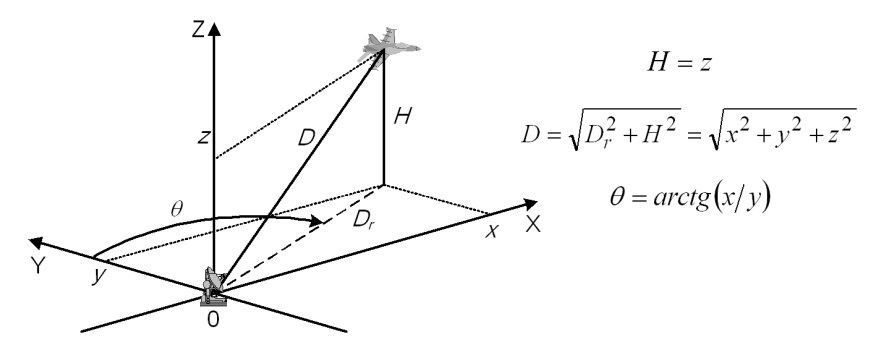

Fig. 1. Relationship of TACAN and ALT measurements to aircraft position.

\section{Closed-loop architecture of INS/TACAN/ALT system}

Integrated positioning system INS/TACAN/ALT can be realized in open-loop or closed-loop configuration. The structure of open-loop INS/TACAN/ALT system has been described in detail in earlier papers by the author [1, 2]. The second paper additionally contains results of simulations which reveal limitations of the open-loop system. Such a system is capable of operating with acceptable errors only for limited time, depending on accuracy of INS. As times of flights are usually relatively short, the open-loop system can still find its applications in aircraft positioning and navigation.

There exists, however, another possible option of integration which seems to be more suitable for systems with low-cost and low-grade inertial systems, especially if they are intended to operate uninterruptedly for longer periods. This alternative option consists in designing INS/TACAN/ALT system with the use of 


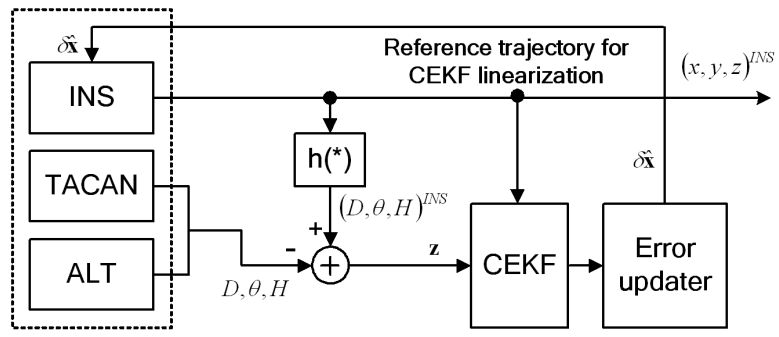

Fig. 2. Closed-loop INS/TACAN/ALT integrated positioning system.

the closed-loop architecture, i.e. with feed-backward INS correction. The structure of such INS/TACAN/ALT system is shown in Fig. 2.

The presented system is tightly coupled as it uses one centralized Kalman filter and treats INS and TACAN/ALT subsystem as sensors [3, 4]. The system consists of three navigation devices and an algorithm of joint data processing, which contains a complementary extended Kalman filter (CEKF) [1-6]. The error updater receives INS errors from CEKF, accumulates them and stores the results. These accumulated errors are subsequently fed to the inertial system to correct INS position, velocity and attitude internally. Thus, the INS output is also the output of the whole positioning system. As the Kalman filter processes already corrected data and estimates only residual INS errors remained after the last correction, INS errors are kept at a relatively low and constant level.

The CEKF filter of the closed-loop system differs from the CEKF designed for the open-loop INS/TACAN/ALT $[1,2,7]$. The discrete dynamics model, on which CEKF is based, is an extension of the standard dynamics model and it includes an additional vector of deterministic inputs $\delta \hat{\boldsymbol{x}}$. The $\delta \hat{\boldsymbol{x}}$ vector has the same size as the state vector $\boldsymbol{x}$, to be estimated via CEKF, and it contains accumulated INS errors from the error updater. The equation of discrete dynamics model is based on the same 8-state model of INS errors as in the open-loop INS/TACAN/ALT system, described in detail in an earlier paper by the author [1] and it has the following form:

$$
\underbrace{\left[\begin{array}{c}
\delta N(k+1) \\
\delta \nu_{\mathrm{N}}(k+1) \\
\phi_{\mathrm{E}}(k+1) \\
\delta E(k+1) \\
\delta \nu_{\mathrm{E}}(k+1) \\
\phi_{\mathrm{N}}(k+1) \\
\delta D(k+1) \\
\delta \nu_{\mathrm{D}}(k+1)
\end{array}\right]}_{\boldsymbol{x}(k+1)}=\underbrace{\left[\begin{array}{cccccccc}
1 & T & 0 & 0 & 0 & 0 & 0 & 0 \\
0 & 1 & g T & 0 & 0 & 0 & 0 & 0 \\
0 & -T / R & 1 & 0 & 0 & 0 & 0 & 0 \\
0 & 0 & 0 & 1 & T & 0 & 0 & 0 \\
0 & 0 & 0 & 0 & 1 & -g T & 0 & 0 \\
0 & 0 & 0 & 0 & T / R & 1 & 0 & 0 \\
0 & 0 & 0 & 0 & 0 & 0 & 1 & T \\
0 & 0 & 0 & 0 & 0 & 0 & 0 & 1
\end{array}\right]}_{\boldsymbol{\Phi}(k+1, k)} \underbrace{\left[\begin{array}{c}
\delta N(k) \\
\delta \nu_{\mathrm{N}}(k) \\
\phi_{\mathrm{E}}(k) \\
\delta E(k) \\
\delta \nu_{\mathrm{E}}(k) \\
\phi_{\mathrm{N}}(k) \\
\delta D(k) \\
\delta \nu_{\mathrm{D}}(k)
\end{array}\right]}_{\boldsymbol{x}(k)}
$$




$$
+\underbrace{\left[\begin{array}{c}
w_{\mathrm{N}}(k) \\
w_{\nu \mathrm{N}}(k) \\
w_{\phi \mathrm{E}}(k) \\
w_{\mathrm{E}}(k) \\
w_{\nu \mathrm{E}}(k) \\
w_{\phi \mathrm{N}}(k) \\
w_{\mathrm{D}}(k) \\
w_{\nu \mathrm{D}}(k)
\end{array}\right]}_{\boldsymbol{w}(k)}-\underbrace{\left[\begin{array}{c}
\delta \hat{N}(k) \\
\delta \hat{\nu}_{\mathrm{N}}(k) \\
\hat{\phi}_{\mathrm{E}} N(k) \\
\delta \hat{E}(k) \\
\delta \hat{\nu}_{\mathrm{E}}(k) \\
\hat{\phi}_{\mathrm{N}}(k) \\
\delta \hat{D}(k) \\
\delta \hat{\nu}_{\mathrm{D}}(k)
\end{array}\right]}_{\delta \hat{\boldsymbol{x}}(k)}
$$

where $\boldsymbol{x}$ - state vector, $\boldsymbol{w}$ - vector of random process disturbances, $\delta \hat{\boldsymbol{x}}$ - vector of deterministic inputs (INS corrections), $\boldsymbol{\Phi}$ - state transition matrix, $\delta N, \delta E, \delta D$ - INS position error along North, East and Down axes $[\mathrm{m}], \delta \nu_{\mathrm{N}}, \delta \nu_{\mathrm{E}}, \delta \nu_{\mathrm{D}}-\mathrm{INS}$ velocity error along North, East and Down axes $[\mathrm{m} / \mathrm{s}], \phi_{\mathrm{E}}, \phi_{\mathrm{N}}$ - INS attitude error around East and North axes [rad], $w_{\mathrm{N}}, w_{\nu \mathrm{N}}, w_{\phi \mathrm{E}}, w_{\mathrm{E}}, w_{\nu \mathrm{E}}, w_{\phi \mathrm{N}}, w_{\mathrm{D}}, w_{\nu \mathrm{D}}, w_{\mathrm{b}}, w_{\mathrm{d}}-$ discrete random process disturbances, $\delta \hat{N}, \delta \hat{\nu}_{\mathrm{N}}, \hat{\phi}_{\mathrm{E}}, \delta \hat{E}, \delta \hat{\nu}_{\mathrm{E}}, \hat{\phi}_{\mathrm{N}}, \delta \hat{D}, \delta \nu_{\mathrm{D}}$ - total estimated INS errors (INS corrections), $g$ - gravity acceleration, $R$ - Earth's radius (spherical model assumed), $T$ - sampling interval of discrete model, $k$ index of discrete time.

The observation model of the closed-loop INS/TACAN/ALT system is the same as the model used for its open-loop version. Also, the matrices necessary for CEKF implementation, i.e. the covariance matrix of discrete process disturbances $\boldsymbol{Q}$, the observation (measurement) matrix $\boldsymbol{H}$ and the covariance matrix of measurement errors $\boldsymbol{R}$ are similar to the respective matrices given in the earlier paper [1], and therefore they are not presented here.

The CEKF contains one initialization and a series of recursive steps of prediction (time update) and correction (measurement update). The equations of filter and error updater are as follows:

initialization:

$$
\hat{\boldsymbol{x}}(0 \mid 0)=\mathbf{0}, \quad \boldsymbol{P}(0 \mid 0)=\boldsymbol{P}(0), \quad \delta \hat{\boldsymbol{x}}(0)=\mathbf{0},
$$

prediction:

$$
\begin{aligned}
& \hat{\boldsymbol{x}}(k+1 \mid k)=\mathbf{0}, \\
& \boldsymbol{P}(k+1 \mid k)=\boldsymbol{\Phi} \boldsymbol{P}(k \mid k) \boldsymbol{\Phi}^{\mathrm{T}}+\boldsymbol{Q}, \\
& \boldsymbol{H}(k+1)=\left[\begin{array}{cccccccc}
\frac{y^{\mathrm{INS}}}{D^{\mathrm{INS}}} & 0 & 0 & \frac{x^{\mathrm{INS}}}{D^{\mathrm{INS}}} & 0 & 0 & \frac{-z^{\mathrm{INS}}}{D^{\mathrm{INS}}} & 0 \\
\frac{-x^{\mathrm{INS}}}{\left(D_{r}^{\mathrm{INS}}\right)^{2}} & 0 & 0 & \frac{y^{\mathrm{INS}}}{\left(D_{r}^{\mathrm{INS}}\right)^{2}} & 0 & 0 & 0 & 0 \\
0 & 0 & 0 & 0 & 0 & 0 & -1 & 0
\end{array}\right], \\
& \boldsymbol{K}(k+1)=\boldsymbol{P}(k+1 \mid k) \boldsymbol{H}(k+1)^{\mathrm{T}}\left[\boldsymbol{H}(k+1) \boldsymbol{P}(k+1 \mid k) \boldsymbol{H}(k+1)^{\mathrm{T}}+\boldsymbol{R}\right]^{-1},
\end{aligned}
$$
correction: 


$$
\begin{aligned}
& \hat{\boldsymbol{x}}(k+1 \mid k+1)=\boldsymbol{K}(k+1) \boldsymbol{z}(k+1), \\
& \boldsymbol{P}(k+1 \mid k+1)=\boldsymbol{P}(k+1 \mid k)-\boldsymbol{K}(k+1) \boldsymbol{H}(k+1) \boldsymbol{P}(k+1 \mid k),
\end{aligned}
$$

total INS error update:

$$
\delta \hat{\boldsymbol{x}}(k+1)=\delta \hat{\boldsymbol{x}}(k)+\hat{\boldsymbol{x}}(k+1 \mid k+1),
$$

where $\hat{\boldsymbol{x}}(k \mid k), \hat{\boldsymbol{x}}(k+1 \mid k+1)$ - filtered state vector at times $k T$ and $(k+1) T$, $\hat{\boldsymbol{x}}(k+1 \mid k)$ - predicted state vector at a time $(k+1) T, \boldsymbol{z}(k+1)$ - measurement vector at a time $(k+1) T, \boldsymbol{P}(k \mid k), \boldsymbol{P}(k+1 \mid k+1)$ - covariance matrix of filtration errors at times $k T$ and $(k+1) T, \boldsymbol{P}(k+1 \mid k)$ - covariance matrix of prediction errors at a time $(k+1) T, \boldsymbol{K}(k+1)$ - Kalman gains matrix at a time $(k+1) T$.

Use of internal INS correction enables significant simplification of the time update and the measurement update in the CEKF filter. Due to correction of all modelled INS errors in each time step of data processing $k$, the predicted state vector equals zero and does not have to be calculated (Eq. (3)). Consequently, in the measurement update (Eq. (7)), the part proportional to the predicted state vector can be omitted and only the part proportional to the measurement vector has to be calculated.

\section{Simulation results and conclusion}

The designed closed-loop INS/TACAN/ALT system has been tested via computer simulations and their chosen results are compared in Fig. 3 with the results obtained for the open-loop system.


Fig. 3. Comparison of positioning errors of INS/TACAN/ALT systems: (a) open-loop, (b) closed-loop.

The presented results have been obtained for the same low-cost and low-grade INS. Errors along the second horizontal axis (East, $X$-axis) display similar behaviour as the errors along $Y$-axis. From the above results one can conclude that the new INS/TACAN/ALT system is capable of long operation without divergence, whereas its open-loop version can work with acceptable level of errors only for a 
relatively short time. The time of operation of open-loop system can be prolonged with the use of higher quality INS, but it increases the cost of the integrated system as well. Thus, the closed-loop system is a better solution also from the economic point of view. On the other hand, use of feed-backward INS correction requires that the INS is able to accept such correcting signals, which is not always the case, and therefore the closed-loop integration is less flexible than the open-loop one.

\section{Acknowledgments}

This paper is financed from sources for science in the years 2007-2010 in the frame of ordered scientific project PBZ-MNiSW-DBO-04/I/2007.

\section{References}

[1] P. Kaniewski, Mol. Quant. Acoust. 28, 165 (2007).

[2] P. Kaniewski, in: ENC-GNSS 2008 - The European Navigation Conf., Toulouse (France) 2008, DVD.

[3] R.G. Brown, P.Y.C. Hwang, Introduction to Random Signals and Applied Kalman Filtering, Wiley, USA 1997.

[4] M.S. Grewal, L.R. Weill, A.P. Andrews, Global Positioning Systems, Inertial Navigation and Integration, Wiley, USA 2001.

[5] M. Kayton, W.R. Fried, Avionics Navigation Systems, Wiley, USA 1997.

[6] P. Kaniewski, Mol. Quant. Acoust. 28, 151 (2007).

[7] D.H. Titterton, J.L. Weston, Strapdown Inertial Navigation Technology, 2nd ed. AIAA, 2004. 